Published in final edited form as:

Nat Neurosci. 2008 October ; 11(10): 1185-1192. doi:10.1038/nn.2197.

\title{
Links from complex spikes to local plasticity and motor learning in the cerebellum of awake-behaving monkeys
}

\author{
Javier F. Medina ${ }^{1}$ and Stephen G. Lisberger ${ }^{2}$ \\ ${ }^{1}$ Department of Psychology, University of Pennsylvania, Philadelphia, PA 19104 \\ ${ }^{2}$ Howard Hughes Medical Institute, W. M. Keck Foundation Center for Integrative Neuroscience, \\ and Department of Physiology, UCSF, San Francisco, CA 94143
}

\begin{abstract}
The hypothesis of cerebellar learning proposes that complex spikes in Purkinje cells engage mechanisms of plasticity in the cerebellar cortex; in turn, changes in the cerebellum depress the simple spike response of Purkinje cells to a given stimulus and cause the adaptive modification of a motor behavior. Although many elements of this hypothesis have been supported by prior experiments, the links between complex spikes, simple spike plasticity, and behavior have not yet been examined simultaneously during the learning process. We now pioneer a trial-by-trial analysis of Purkinje cell responses in awake-behaving monkeys, with results that strongly favor a causal role for complex spikes in the induction of cerebellar plasticity during a simple motor learning task. We show that the presence of a complex spike on one learning trial is linked to a substantial depression of simple spike responses on the subsequent trial, at a time when behavioral learning is expressed.
\end{abstract}

The cerebellum possesses two input systems that are very different in both anatomy and physiology 1,2. The mossy fiber system originates in many brain regions, and projects to Purkinje cells in the cerebellar cortex through granule cells and their parallel fibers as well as through a number of different inhibitory interneurons. Each Purkinje cell receives inputs from tens of thousands of parallel fibers, resulting in traditional "simple spikes" with spontaneous rates of $100 \mathrm{~s}^{-1}$ or higher. The climbing fiber system originates in the inferior olive. Each Purkinje cell receives massive inputs from just one climbing fiber, resulting in "complex spikes" that have an unusual waveform and occur infrequently, about once per second. The striking differences between the two systems led to the idea that their interplay may create a learning system for movement3-5.

The theory of cerebellar learning is comprised of three separate, but connected, hypotheses. First, climbing fiber inputs are activated when a movement is inaccurate or erroneous6,7. Second, the activation of the climbing fiber input engages mechanisms of plasticity that cause changes in synaptic strength and alter the simple spike responses of Purkinje cells8,9. Third, these changes in cerebellar output lead to adaptive modification of the motor behavior10-14. Each of these three hypotheses has received considerable experimental support, but the theory of cerebellar learning has not been tested as a full entity by examining all three hypotheses simultaneously within the same experiment. In addition, it has been difficult to draw strong conclusions about cause-and-effect links between complex 
spikes, simple spikes, and behavioral learning, because previous work has treated plasticity in the cerebellar cortex as a static process, by measuring the simple spike response of Purkinje cells after learning, at a time when the climbing fiber inputs are no longer activated. A more rigorous test of the cerebellar learning theory demands that we examine the dynamic interaction between complex spikes and the induction of neural and behavioral learning on a trial-by-trial basis during the learning process itself.

We have developed a new approach that tests directly for cause-and-effect links from complex spikes in single trials to changes in simple spike firing, and in turn to behavioral learning in awake, behaving monkeys. While monkeys were actively learning smooth pursuit eye movements 15, we recorded from single Purkinje cells in the floccular complex of the cerebellum - a structure that is only two synapses removed from motoneurons, and that is critical for both the initiation and maintenance of pursuit16,17. We found that the presence or absence of a complex spike on one learning trial determines whether or not the simple spike response of the Purkinje cell will be depressed on the subsequent trial, and we show with the same dataset how complex spike responses during learning are related to changes in simple spike responses that cause behavioral learning.

\section{Results}

Even while investigating simultaneously the full set of linkages among complex spikes, learned changes in simple spike responses, and learning in pursuit behavior, we need to separate the question of how complex spikes during learning lead to changes in simple spike responses and how the changes in simple spike responses lead to changes in behavior. This is because the three variables we measure are linked by mechanisms that operate independently, at different loci, and at different times. We start by considering the linkage between changes in simple spike responses and changes in eye velocity measured at the same time. Then, we evaluate the linkage from complex spikes on learning trials to changes in the simple spike responses recorded on subsequent probe trials. Finally, we show that a complex spike on one trial is associated with a large and specifically-timed decrease of the simple spike response on the next trial.

\section{Simple spike correlates of directional learning in pursuit}

In a typical learning experiment15, a monkey performed 200 learning trials (Figs. 1a, c), each one beginning with target motion to the right at $20 \mathrm{deg} \mathrm{s}^{-1}$. After $250 \mathrm{~ms}$, we introduced an instructive stimulus in the form of a vertical component of target motion at 30 $\operatorname{deg~} \mathrm{s}^{-1}$, so that the target moved up and to the right for $450 \mathrm{~ms}$ before stopping. Starting about $100 \mathrm{~ms}$ after the onset of upward target motion, the monkey began to move his eyes upwards (arrow pointing up and left in Fig. 1c), and then made a large saccade to catch up with the target. There is, in addition, a more subtle difference in the vertical eye velocity traces between the early and late learning trials (Fig. 1c). In the late learning trials (black traces), an upward vertical eye velocity appears just before the onset of upward target motion (arrow pointing down and right). This early upward eye velocity is a learned response because it precedes the instructive stimulus, and because it occurs after but not 
before learning (black versus gray traces in Figs. 1b, d) in occasional probe trials that present purely rightward target motion.

Consider a learning experiment on a Purkinje cell with a rightward on-direction, defined by the direction of pursuit associated with the largest simple spike response before learning; the off-direction was leftward. In pre-learning probe trials (Fig. 2a), the Purkinje cell had a relatively small simple spike response during pure downward pursuit (light blue trace), and had larger and smaller simple spike responses, respectively, during pursuit with a small rightward (purple) or leftward (green) component.

In the learning trials of learning block \#1 (black arrow in left column of Fig. 2b), the target moved downward for $250 \mathrm{~ms}$ and then changed direction abruptly to include a leftward (offdirection) component of motion at $30 \mathrm{deg} \mathrm{s}^{-1}$. After 100 learning trials, occasional probe trials consisting of purely downward target motion (red arrow) evoked a leftward component of smooth eye motion that began about $200 \mathrm{~ms}$ after the onset of target motion, anticipating the change of target direction that would have occurred $50 \mathrm{~ms}$ later in the learning trials (red trace, Fig. 2b). In the first learning block, the change in target direction was randomly selected to be either in the on- or off-direction of the Purkinje cell; we always proceeded to a second learning block that evoked learning in the opposite direction (Fig. 2c). For the example Purkinje cell, behavioral learning ("Eye velocity" column of Figs. 2b, c) was modeled by a remarkably similar, reciprocal change in simple spike firing rate for both directions of learning ("SS firing rate" column of Figs. 2b, c). In subsequent figures we capture the learned eye velocity and simple spike firing rate as the millisecond-bymillisecond difference between the average response in the learning block minus the prelearning block.

A large majority of the Purkinje cells in our sample showed learned changes in simple spike firing that had the same shape and direction as the changes in eye velocity (Figs. 3c, e, n=49, "group 1"). For group 1 Purkinje cells, the learned change in eye velocity (black traces in Fig. 3c) lagged the learned change in simple spike firing (colored traces in Fig. 3c) by a small time delay of 36 and $28 \mathrm{~ms}$ for on-direction and off-direction learning. For the group 1 Purkinje cells, the average peak-to-peak changes in simple spike firing and eye velocity were approximately 30 spikes s ${ }^{-1}$ and $12 \mathrm{deg} \mathrm{s}^{-1}$, for a ratio of 2.5 spikes s$^{-1}$ per deg s $\mathrm{s}^{-1}$.

For a minority of our sample, the learned changes in simple spike firing and eye velocity were not reciprocal (Figs. 3d, f, n=11, "group 2"). The most notable deviation of the learned simple spike responses of group 2 Purkinje cells was a delayed increase in firing for offdirection learning. The difference between the learned responses of group 1 and 2 Purkinje cells could not be attributed to differences in the learned eye velocities (Figs. 3e, f). The increases in simple spike firing of group 2 Purkinje cells modeled the positive portion of the learned eye acceleration (black traces in Fig. 3d), that is when the eyes are speeding up in the on-direction or slowing down in the off-direction. The learned simple spike responses of group 1 and 2 Purkinje cells divide neatly in terms of relationships to learned eye velocity versus acceleration. However, the same clean division was not observed in the pre-learning block of trials used to measure the directional selectivity of each Purkinje cell: during prelearning trials in either the on- or off-direction, all Purkinje cells in both groups showed an 
eye velocity component of firing during steady-state pursuit and many in each group showed a transient during eye acceleration at the initiation of pursuit (see also18,19).

\section{From instructive complex spikes to simple spike learning}

Consistent with previous work, we found complex spike responses to image motion in the off-direction20,21, i.e. when the eyes are moving more slowly than the target in the offdirection, or faster than the target in the on-direction. During off-direction learning trials, however, group 1 and group 2 Purkinje cells differed in the probability of emitting a complex spike (Figs. 3a, b, red traces). The probability of a complex spike in the interval $75-175 \mathrm{~ms}$ after the change in target direction increased to an average of 0.5 in group 1 versus 0.2 in group 2 . Because in our experiments eye (and image) velocity was essentially the same on trials with and without a complex spike (Supplementary Fig. 1), the occurrence of a complex spike on any particular learning trial reflects its stochastic nature, and not a large trial-by-trial difference in the magnitude of image motion caused by the instructive change in target direction. During on-direction learning trials, complex spike responses showed relatively little modulation (Figs. 3a, b, blue traces), except for a prominent peak that will be considered later because it occurred $125 \mathrm{~ms}$ after the offset of target motion (more than $800 \mathrm{~ms}$ after the onset of target motion).

To test quantitatively the relationship between off-direction learning in the simple spike firing of each Purkinje cell and the probability of emitting a complex spike in response to the learning stimulus, it is necessary to appreciate the relative timings of the learned eye velocity and simple spike firing in probe trials, and the complex spike responses in learning trials. Complex spike responses are time-locked to the instructive visual stimulus. They occur $\sim 125 \mathrm{~ms}$ after the change in target direction20,21 and are measured from 75 to 175 ms after the change in target direction in learning trials (gray areas in Figs. 3a, b). The learned eye velocity and simple spike firing anticipate the change in target direction 15 . They start 50-100 ms before the time of the change in target direction in learning trials (leftmost vertical dashed lines in Fig 3) and can be measured in either learning or probe trials, in the interval from $50 \mathrm{~ms}$ before to $50 \mathrm{~ms}$ after the time when the change in target direction occurs in the learning trials (gray areas in Figs. 3c, e).

Across Purkinje cells, the probability of a complex spike during learning was a good predictor of the size and direction of the learned change in simple spike firing in each individual Purkinje cell (Fig. 4). For learning in the off-direction, group 1 Purkinje cells had a high probability of emitting a complex spike in the learning trials and showed large learned decreases in simple spike firing in probe trials (filled red circles); group 2 Purkinje cells were less likely to emit a complex spike during learning and showed learned increases, rather than decreases, in simple spike firing (open red circles). For learning in the ondirection, neither group of Purkinje cells emitted very many complex spikes in the learning trials and both showed learned increases in simple spike firing during the probe trials (open and filled blue circles). Averaging the response measures in bins (black symbols) indicates that the probability of a complex spike had to at least triple from baseline to be consistently associated with a learned decrease in simple spike response. 
If complex spike-instructed cellular changes were the only plasticity mechanism operating in the cerebellar cortex, then we would have expected zero learning in simple spike firing when complex spike probability was at baseline levels. This prediction is contradicted by the data in Figure 4. When the probability of a complex spike is at baseline levels (vertical dashed line), and thus cannot possibly be signaling "errors", there are consistent learned increases in simple spike responses for on-direction learning. Therefore, we suggest that complex spikeinstructed cellular changes cannot be the only plasticity mechanism at work during pursuit learning.

The tight correlation between the probability of a complex spike and the amount of neural and behavioral learning is emphasized by comparison of the time courses of different response measures during off-direction learning for group 1 versus group 2 Purkinje cells. For all Purkinje cells, the learned eye velocity (Fig. 5a) changes quickly in the first 3 sets of 10 learning trials, and then reaches an asymptote. Considering each set of learning trials separately, simple spike firing (Fig. 5b) decreased the most at times when the complex spike probability (Fig. 5c) was higher than 3x the normal baseline level of about 0.1 in a $100 \mathrm{~ms}$ bin, for example in the first 3 sets of 10 learning trials for group 1 Purkinje cells. At times when the complex spike probability was lower than 0.3 during the learning trials, including all times for group 2 Purkinje cells, we observed learned increases in simple spike responses.

\section{Simple spike learning after single complex spikes}

The results presented so far demonstrate impressive correlations between the learned changes in simple spike firing and eye velocity, and between the complex spike activity in learning trials and the subsequent changes in the simple spike firing of each Purkinje cell. Still, these data treat learning as a static process, comparing average responses across multiple trials that occurred in different phases of the learning process. To move from correlation to cause-and-effect using the same dataset, we now examine changes in simple spike responses between two successive learning trials. If the presence (but not the absence) of a complex spike response to the instructive stimulus on one trial were associated with a reduction in simple spike firing rate at the correct time on the subsequent trial, then we could conclude that there is a tight link between complex spike responses and depression of simple spike responses, during behavioral learning.

We looked for complex spike-linked trial-over-trial changes in simple spike responses in the first 50 trials of off-direction learning, where most of the learning takes place and where the learning stimulus evokes complex spike responses with a high probability, at least for group 1 Purkinje cells (Figs. 3-5). As described in the Methods, we evaluated pairs of successive learning trials, characterizing each according to the presence or absence of a complex spike in the interval from 75 to $175 \mathrm{~ms}$ after the change in target direction. We retained pairs that lacked a complex spike in both trials of the pair (" $0-0$ ") or that had a complex spike only in the first trial of the pair ("1-0"), and computed the difference in simple spike firing between the first and second trial of the pair (Fig. 6b). We looked for depression of simple spike responses at the same time when learning normally is expressed, just before the time of the change in target direction that serves as an instructive stimulus. When there was a complex 
spike in the first trial (red trace, "1-0" pairs), the trial-over-trial change in firing in the simple spike analysis interval was negative, relative to both the " $0-0$ " pairs (blue trace) and the average of 200 random draws of consecutive learning trials (black trace). The statistical significance of the trial-over-trial reduction in simple spike firing for "1-0" pairs is supported by the fact that the red trace lies outside the \pm 2 SD envelope from the randomdraw analysis (gray area) for almost the entire analysis window, and almost exclusively within the analysis window. In contrast, the trial-over-trial change in simple spike firing for the " $0-0$ " trials was very similar to the random-draw average. Figure 6a demonstrates that the depression of simple spike firing between the first and second trials of "1-0" pairs was due entirely to lower than normal simple spike activity on the second trial of the pair (purple trace). Note that the trial-over-trial depression of simple spike activity is not related to the well-known post-complex spike pause in simple spike firing6,22: the latter occurs after the complex spike on the same trial, whereas the former occurs before the time of the complex spike, and on the subsequent trial.

The presence of a complex spike on one trial also affected the learned eye velocity on the subsequent trial, although the effect was small and did not quite reach statistical significance: the red trace in Figure $6 \mathrm{c}$ stays within the \pm 2 SD envelope from the randomdraw analysis. The changes in simple spike firing and eye velocity for the "1-0" pairs were approximately 12 spikes s ${ }^{-1}$ and $0.8 \mathrm{deg} \mathrm{s}^{-1}$, yielding a ratio of 15 spikes s$^{-1}$ per deg s $\mathrm{s}^{-1}$. The meaning of this eye velocity sensitivity ratio, which is 6 times larger than the one measured from the data in Figure 3, is treated quantitatively in the Supplementary Appendix. Note that Figure 6 presents analyses performed on individual Purkinje cells and then averaged across a population of 13 Purkinje cells. Supplementary Figure 2 shows that the trial-over-trial reduction of simple spike firing in the "1-0" pairs is a feature of most individual Purkinje cells and indeed of most individual pairs of trials.

Figure 6 verifies a key prediction of the cerebellar learning theory on data obtained during motor learning in a behaving primate: a complex spike in one trial is linked to a measurable and properly timed depression of the simple spike response on the subsequent trial. The depression of simple spike activity peaks approximately $100 \mathrm{~ms}$ before the time of the complex spike response on the prior trial, which is consistent with the frequently-discussed requirement of an "eligibility trace" for cerebellar LTD so that a complex spike can affect the synaptic inputs that were active $100 \mathrm{~ms}$ earlier23-26.

An independent confirmation of the trial-over-trial effect of a single complex spike comes from analysis of an unanticipated sharp increase in the probability of complex spike responses just after the offset of target motion for on-direction learning trials (Fig. 7b). At this time, smooth eye velocity is in the on-direction of the Purkinje cell (for simple spike responses), and off-direction image motion (the main stimulus for complex spike responses 20,21 ) occurs because the target is suddenly stationary while the eyes keep moving (arrow in Fig. 7a).

The presence of a complex spike after the offset of target motion in one trial caused a depression of the simple spike response and the eye velocity on the subsequent trial. The depression of simple spike firing (Fig. 7d) is statistically significant and has a similar time- 
course to that shown in Figure $6 \mathrm{~b}$; it starts $150 \mathrm{~ms}$ before and peaks $100 \mathrm{~ms}$ before the offset of target motion. The companion trial-over-trial decrease in eye velocity has two statistically-significant negative peaks but returns to zero in the middle of the analysis interval (Fig. 7e). In Figures 7d and e, the peak trial-over-trial changes in simple spike firing rate and eye velocity are 9 spikes s $\mathrm{s}^{-1}$ and $0.6 \mathrm{deg} \mathrm{s}^{-1}$ for a ratio that again is 15 spikes s $\mathrm{s}^{-1}$ per $\operatorname{deg} \mathrm{s}^{-1}$.

Finally, we ask whether the complex spike response at the offset of on-direction target motion is linked to any learning expressed as a difference in simple spike and eye velocity responses before versus after 100 learning trials. Surprisingly, it is, even though the offset of target motion was not intended as a learning stimulus. Difference traces computed each millisecond as the average response in late learning trials minus that in early learning trials reveal increases in both simple spike firing (Fig. 7f, red trace) and eye velocity (black trace) at times that are commensurate with the time of the stimuli that led to complex spike response: both reach peaks near the time of target motion offset ( $\mathrm{t}=700 \mathrm{~ms})$. As in Figure 3c, the learned eye velocity lags the learned simple spike firing by a few milliseconds. Note that it is difficult to interpret the noise in the traces of Figure $7 \mathrm{f}$ at times between 400 and $600 \mathrm{~ms}$ after the onset of target motion because we were not able to remove the effects of saccades in this interval from the simple spike firing.

Why do we see a local depression of the simple spike response on the trial following a complex spike, but learned increases in simple spike responses and eye velocity at the offset of on-direction target motion after 100 learning trials? The basis for this seemingly contradictory result lies in Figure $7 \mathrm{~g}$. When we plot the magnitude of the learned change in simple spike firing as a function of the complex spike probability 75-175 ms after the offset of on-direction target motion in the learning trials, the data for the individual Purkinje cells (blue symbols) follow the same relationship found during off-direction learning in Figure 4 (gray symbols). Thus, all our data are consistent with the notion that learning will be associated with an increase in simple spike firing over the course of a block of learning trials unless complex spike probability is more than $3 x$ baseline levels, in which case the simple spike responses during the learned behavior will decrease.

\section{Discussion}

To understand the neural basis of motor learning, we must examine how learning stimuli, neural plasticity, and behavior interact at the systems level. We need to identify all the loci of learning within a neural system and establish at each site the linkages among three key elements: properly timed instructive signals that indicate errors and activate mechanisms of plasticity; learning-related changes in the firing rate of neurons that provide the output from that site; and the adaptive modification of the behavior. In contrast to prior studies, which restricted themselves to one or two of these links at a time, the major contribution of our study is to demonstrate all three links with a single set of neural and behavioral data obtained by recording cerebellar responses before, during, and after learning of smooth pursuit eye movements in awake monkeys. 
By evaluating both the trial-over-trial changes that take place during the learning process, and the static state of the system before and after learning, we were able to evaluate local complex spike-instructed mechanisms of plasticity in the cerebellar cortex within the larger context of system-level changes in the neural circuits that mediate motor learning in pursuit. Our trial-over-trial analysis establishes that a complex spike error signal on one learning trial is linked to depression of the same Purkinje cell's simple spike activity on the subsequent trial. The depression is present selectively from 250 to $100 \mathrm{~ms}$ before the time in the trial when the complex spike had occurred, consistent with the timing of learning in pursuit15, and with a number of prior reports concluding that a complex spike must cause long-term depression (LTD) in parallel fiber synapses that were active 100-200 ms before the complex spike23-26. The depression has the same temporal characteristics and amplitude in two different and independent sets of learning trials, providing strong statistical support for our results. We think that the trial-over-trial changes in simple spike firing are a consequence of plasticity mechanisms within the cerebellar cortex, demonstrated here during the learning process in awake, behaving animals for the first time.

"Cerebellar learning" has often been considered synonymous with "motor learning", but it is important to remember that a complex circuit of neurons generates pursuit and that a whole concert of plastic changes is likely needed at multiple sites in the circuit to cause stable behavioral learning10-12,14. Even though the trial-by-trial analysis indicates that complex spike-instructed depression of simple spike activity is one of the local mechanisms contributing to pursuit learning, there must be other mechanisms of plasticity. For example, we found that 100 learning trials cause a learned change in simple spike responses that is related, in an unexpected way that was not predicted by the cerebellar learning theory, to the probability of complex spike responses during those 100 learning trials. If the complex spike responses of a Purkinje cell during the learning trials are at baseline levels, or even up to twice baseline levels, then behavioral learning is linked to a learned increase in the simple spike firing of that particular Purkinje cell. This finding complements, and does not contradict, our trial-over-trial finding of complex spike-linked depression. It suggests that there is a competition where different local mechanisms of potentiation and depression are balanced when the probability of a complex spike is about three times control levels. The interplay of multiple plasticity mechanisms is not entirely unexpected. There are examples of cellular plasticity at many sites in the cerebellar cortex9,27-29, and previous work has indicated multiple sites of plasticity even in simple forms of cerebellar-dependent motor learning like adaptation of the vestibulo-ocular reflex30,31 and classical delay conditioning of eyelid responses 32,33

Comparison of eye velocity and simple spike responses before versus after learning showed, as others have shown before34-37, that learned simple spike responses could drive the learned eye movement. For this to be a causal link, however, changes in simple spike firing must cause the changes in behavior, and not vice versa. Because Purkinje cells normally fire in relationship to smooth eye velocity 38 , and because eye velocity changes during our learning task, at least the changes in simple spike firing of group 1 Purkinje cells could result simply from mossy fiber inputs that report the kinematic parameters of the current eye movement39. We think that this scenario is unlikely. First, there is a disynaptic connection 
from floccular Purkinje cells to extraocular motoneurons40, and electrical stimulation in the floccular complex causes smooth eye movement with a latency of $10 \mathrm{~ms} 30,41$, making it difficult to argue that a change in simple spike firing would not drive a change in eye movement. Second, as shown in Figure 3b, eye velocity lags the simple spike response of group 1 Purkinje cells by more than 10 milliseconds. Therefore, we think that the simple spike correlates of behavioral learning are primary learned responses that are driving, rather than responding to, the learned behavior.

We also think that the complex spike-linked, trial-over-trial depression in simple spike firing is much too large to be a consequence of cerebellar inputs that report trial-over-trial changes in eye velocity. Purkinje cells in our sample had an average eye velocity sensitivity of approximately 2.5 spikes $\mathrm{s}^{-1}$ per deg s $\mathrm{s}^{-1}$ measured during the expression of pursuit learning. Given this measurement, and the fact that the trial-over-trial change in eye velocity had a peak of $0.8 \mathrm{deg} \mathrm{s}^{-1}$, the trial-over-trial depression of simple spike firing rate would be 0.8 times 2.5 , or 2 spikes $\mathrm{s}^{-1}$ if Purkinje cells were simply responding to the kinematics of the trial-over-trial change in eye movement. In fact, the trial-over-trial depression of simple spike firing was 12 spikes s $^{-1}$. Therefore, the trial-over-trial depression of simple spike firing cannot be simply reporting the behavioral response. The Supplementary Appendix provides an explanation for the surprisingly large trial-over-trial changes in simple spike firing in terms of what may be happening across the full population of Purkinje cells rather than just the Purkinje cell under study.

The highly stochastic nature of complex spike activity during learning paints a picture that contrasts with the view that motor learning occurs as a series of gradual steps whose size is proportional to the size of the error on a particular trial42-45 (but see46). A complex spike occurs at most on half of learning trials, but is linked to a large depression of simple spike firing on the subsequent trial. Perhaps the contribution of individual Purkinje cells to motor learning is a random walk with larger steps in the learning direction than the reverse. It remains to be seen whether this also is true at the population or behavioral levels, a question that may ultimately be answered by applying properly contrived quantitative methods to both behavioral and neural data47. It also will be informative to determine whether a dynamic trial-by-trial interplay of potentiation and depression is uncovered when in vitro experiments use plasticity protocols that mimic the stochastic nature of the in vivo complex spike responses.

Our paper is the first to examine trial-over-trial neural changes in awake animals actively engaged in a motor learning task, allowing us to make direct estimates of local cellular plasticity mechanisms engaged during the dynamic learning process. By also evaluating how a block of learning trials changes the state of cerebellar and behavioral outputs, we have begun to assemble an understanding of the interaction between cellular and systems-level mechanisms of motor learning. The approach we have initiated is built upon the foundation provided by earlier studies done in anesthetized or paralyzed animals 48 , but goes further because it can assess the relationship between plasticity and behavior simultaneously, and because it can be deployed during learning with natural sensory stimuli. We have found evidence that strongly supports the predictions of the cerebellar learning theory3-5, while 
also reminding us that learning in any single behavior is likely to involve many cellular mechanisms at a variety of loci within the full neural circuit.

\section{Methods}

\section{Animal preparation}

We recorded the responses of single Purkinje cells in 2 awake, behaving rhesus monkeys. Monkeys had been instrumented with an implanted head-holder to allow us to immobilize the head in a way that did not cause distress 49 , a coil of wire on one eye to allow use of the search coil system to monitor eye movement 49 , and a recording cylinder that allowed us to gain access to the floccular complex of the cerebellum with metal microelectrodes 19 . These devices were implanted in surgical procedures that used isofluorane as an anesthetic and were conducted with sterile procedure. Monkeys received several days of analgesics following each surgery. All procedures had been approved in advance by the Institutional Animal Care and Use Committee at UCSF and were in accordance with the NIH Guide for the Care and Use of Laboratory Animals.

\section{Pursuit task}

Monkeys were trained to fixate and track a target presented on a screen in front of them in exchange for fluid rewards. Targets were created by imaging the beam from a fiber optic light source and reflecting it off a pair of computer-controlled, moveable mirror galvanometers onto the back of a tangent screen. Experiments were conducted as a series of trials where each trial started with a brief fixation period $(500-1000 \mathrm{~ms})$, then provided a sequence of target motions, and terminated by holding the target stationary for $500 \mathrm{~ms}$. The onset of target motion followed standard a step/ramp trajectory, with the size of the step adjusted to minimize the occurrence of saccades. Monkeys were required to fixate on the target when it appeared, track it as best they could as it moved during the trial, and fixate it again at the end of its motion, keeping eye position within a $2 \times 2 \mathrm{deg}$ window centered on the moving target. Successful performance on a trial was rewarded with a droplet of fluid. Reward contingencies were suspended for $250 \mathrm{~ms}$ whenever the target underwent a change in position or velocity, to ensure that the monkey was not punished for the inescapable latencies of the visual system. To generate eye velocity traces we used an analog circuit to differentiate the eye position records for frequencies below $25 \mathrm{~Hz}$, while filtering out higher frequencies. Analog signals related to horizontal and vertical target position, eye position, and eye velocity were sampled and digitized at $1 \mathrm{kHz}$ per channel.

Experiments were conducted as three separate blocks. The baseline block comprised approximately 100 trials in which the target moved at constant speed ( $\left.20 \mathrm{deg} \mathrm{s}^{-1}\right)$ for $750 \mathrm{~ms}$ in one of eight directions. It was followed by two learning blocks, each one comprising approximately 300 trials. Most of the trials in a learning block were "learning" trials that started with $250 \mathrm{~ms}$ of target motion at $20 \mathrm{deg} \mathrm{s}^{-1}$ in a direction orthogonal to the ondirection of the Purkinje cell under study. Then, the target provided an instructional change in direction by acquiring a component of motion at $30 \mathrm{deg} \mathrm{s}^{-1}$ in the on- or off-direction of the Purkinje cell and continuing to move for an additional $450 \mathrm{~ms}$. The instructional change in target direction was the same in all learning trials of a given learning block, and was in 
the on- or off-direction of the Purkinje cell under study in the two learning blocks. To assess the extent of learning, approximately 20-30 "probe" trials were randomly interleaved among the learning trials: in probes, the target began to move as in the learning trials but did not change direction.

\section{Single-unit recording}

Platinum-iridium micro-electrodes manufactured in our laboratory were introduced daily and driven through the cerebral cortex and the tentorium of the cerebellum until they reached the floccular complex. Entrance in to the cerebellum was heralded by a large increase in the general level of spontaneous activity and the presence of complex spike responses. Entrance into the floccular complex was signaled by the presence of eye movement-related activity and the sound of Purkinje cells modulating strongly as the monkey tracked sinusoidal target motion along the horizontal or vertical axis. Extracellular activity was passed through a standard head stage, amplified, filtered (bandpass, $100 \mathrm{~Hz}$ to $10 \mathrm{kHz}$ ), digitized at $25 \mathrm{KHz}$, and stored in the computer for further processing. Because it was challenging to keep the complex spike responses isolated throughout a full experiment, we were able to include only 17 Purkinje cells in our analysis of complex spike responses. However, we were able to document the learned simple spike responses of 60 Purkinje cells that were held through the full learning protocol, with each Purkinje cell identified by the presence of a clear complex spike response for part of the recording.

\section{Data analysis}

Digitized eye velocity traces from individual trials were displayed on the computer screen, and all saccades were excised manually. Then, we used a software window discriminator to go through each trial in the data set and mark simple spikes and complex spikes by hand. The simple spike response was transformed into a firing rate using the reciprocal interval algorithm50. Complex spike responses were accumulated for many trials in bins with widths of $100 \mathrm{~ms}$ and then converted to the probability of a complex spike in a particular bin. We also introduce a new trial-by-trial analysis inspired partly by a prior paper 47 . We characterized each pair of successive learning trials according to the presence or absence of a complex spike in the interval from 75 to $175 \mathrm{~ms}$ after the change in target direction. We retained pairs that lacked a complex spike in both trials of the pair ("0-0") or that had a complex spike only in the first trial of the pair ("1-0"), but only if the learning trial immediately before the pair had lacked a complex spike. The requirement for the absence of a complex spike in a learning trial immediately before the pair reduced the total number of sequences that met all criteria, but was necessary to establish a consistent history of visual stimulus and complex spike response for every sequence included in our analysis. We then computed the change in simple spike firing rate and in the eye velocity between the trials of each "1-0" and " $0-0$ " pair. For the data presented in Figures 6 and 7, the trial-over-trial changes in simple spike firing rate and eye velocity were averaged separately for each Purkinje cell as long as there were at least four " $1-0$ " and four " $0-0$ " pairs; the averages then were pooled to provide a grand average of trial-by-trial changes across Purkinje cells. To assess statistical significance, we generated a series of 100 "control" distributions of trialover-trial changes for each Purkinje cell separately. Each distribution contained 200 random sequences of two consecutive learning trials drawn from all available sequences (including 
"0-0", "0-1", "1-0" and "1-1" pairs). The mean of the "control" distributions represents the average trial-over-trial change that we would expect for each Purkinje cell over two consecutive learning trials, regardless of whether there was a complex spike in any of the trials or not. The standard deviation of the "control" distributions allows us to assess statistical significance by asking whether the measured trial-over-trial changes for " $1-0$ " and "0-0" pairs lie outside the $95 \%$ confidence intervals.

\section{Supplementary Material}

Refer to Web version on PubMed Central for supplementary material.

\section{Acknowledgments}

Research supported by the Howard Hughes Medical Institute and by NIH grant P50 MH77970

\section{References}

1. Eccles, JC.; Ito, M.; Szentágothai, J. The Cerebellum as a Neuronal Machine. Springer-Verlag; Berlin, New York: 1967.

2. Ito, M. The cerebellum and neural control. Raven Press; New York: 1984.

3. Albus JS. A theory of cerebellar function. Mathematical Bioscience. 1971; 10:25-61.

4. Marr D. A theory of cerebellar cortex. Journal of Physiology. 1969; 202:437-70. [PubMed: 5784296]

5. Ito M. Neural design of the cerebellar motor control system. Brain Res. 1972; 40:81-4. [PubMed: 4338265]

6. Simpson JI, Wylie DR, de Zeeuw CI. On climbing fiber signals and their consequence(s). Behav.Brain Sci. 1996; 19:384-398.

7. de Zeeuw CI, et al. Microcircuitry and function of the inferior olive. Trends Neurosci. 1998; 21:391-400. [PubMed: 9735947]

8. Ito M. Cerebellar long-term depression: characterization, signal transduction, and functional roles. Physiol Rev. 2001; 81:1143-1195. [PubMed: 11427694]

9. Hansel C, Linden DJ, D'Angelo E. Beyond parallel fiber LTD: the diversity of synaptic and nonsynaptic plasticity in the cerebellum. Nat.Neurosci. 2001; 4:467-475. [PubMed: 11319554]

10. De Zeeuw CI, Yeo CH. Time and tide in cerebellar memory formation. Curr Opin Neurobiol. 2005; 15:667-74. [PubMed: 16271462]

11. Boyden ES, Katoh A, Raymond JL. Cerebellum-dependent learning: the role of multiple plasticity mechanisms. Annu Rev Neurosci. 2004; 27:581-609. [PubMed: 15217344]

12. Carey M, Lisberger S. Embarrassed, but not depressed: eye opening lessons for cerebellar learning. Neuron. 2002; 35:223-6. [PubMed: 12160741]

13. Ito M. Mechanisms of motor learning in the cerebellum. Brain Res. 2000; 886:237-245. [PubMed: 11119699]

14. Raymond JL, Lisberger SG, Mauk MD. The cerebellum: a neuronal learning machine? Science. 1996; 272:1126-1131. [PubMed: 8638157]

15. Medina JF, Carey MR, Lisberger SG. The representation of time for motor learning. Neuron. 2005; 45:157-67. [PubMed: 15629710]

16. Robinson FR, Fuchs AF. The role of the cerebellum in voluntary eye movements. Annu Rev Neurosci. 2001; 24:981-1004. [PubMed: 11520925]

17. Lisberger SG, Morris EJ, Tychsen L. Visual motion processing and sensory-motor integration for smooth pursuit eye movements. Annu.Rev.Neurosci. 1987; 10:97-129. [PubMed: 3551767] 
18. Stone LS, Lisberger SG. Visual responses of Purkinje cells in the cerebellar flocculus during smooth-pursuit eye movements in monkeys. I. Simple spikes. J Neurophysiol. 1990; 63:1241-61. [PubMed: 2358872]

19. Medina JF, Lisberger SG. Variation, signal, and noise in cerebellar sensory-motor processing for smooth-pursuit eye movements. J Neurosci. 2007; 27:6832-42. [PubMed: 17581971]

20. Stone LS, Lisberger SG. Detection of tracking errors by visual climbing fiber inputs to monkey cerebellar flocculus during pursuit eye movements. Neurosci Lett. 1986; 72:163-8. [PubMed: 3101005]

21. Stone LS, Lisberger SG. Visual responses of Purkinje cells in the cerebellar flocculus during smooth-pursuit eye movements in monkeys. II. Complex spikes. J Neurophysiol. 1990; 63:126275. [PubMed: 2358873]

22. Sato Y, Miura A, Fushiki H, Kawasaki T. Short-term modulation of cerebellar Purkinje cell activity after spontaneous climbing fiber input. J.Neurophysiol. 1992; 68:2051-2062. [PubMed: 1491256]

23. Raymond JL, Lisberger SG. Neural learning rules for the vestibulo-ocular reflex. J.Neurosci. 1998; 18:9112-9129. [PubMed: 9787014]

24. Doi T, Kuroda S, Michikawa T, Kawato M. Inositol 1,4,5-trisphosphate-dependent Ca2+ threshold dynamics detect spike timing in cerebellar Purkinje cells. J Neurosci. 2005; 25:950-61. [PubMed: 15673676]

25. Wang SS, Denk W, Hausser M. Coincidence detection in single dendritic spines mediated by calcium release. Nat Neurosci. 2000; 3:1266-73. [PubMed: 11100147]

26. Houk JC, Alford S. Computational significance of the cellular mechanisms for synaptic plasticity in Purkinje cells. Behavioral and Brain Sciences. 1996; 19:457.

27. Jorntell H, Hansel C. Synaptic memories upside down: bidirectional plasticity at cerebellar parallel fiber-Purkinje cell synapses. Neuron. 2006; 52:227-38. [PubMed: 17046686]

28. Mittmann W, Hausser M. Linking synaptic plasticity and spike output at excitatory and inhibitory synapses onto cerebellar Purkinje cells. J Neurosci. 2007; 27:5559-70. [PubMed: 17522301]

29. Kano M, Rexhausen U, Dreessen J, Konnerth A. Synaptic excitation produces a long-lasting rebound potentiation of inhibitory synaptic signals in cerebellar Purkinje cells. Nature. 1992; 356:601-4. [PubMed: 1313949]

30. Lisberger SG. Neural basis for motor learning in the vestibuloocular reflex of primates. III. Computational and behavioral analysis of the sites of learning. Journal of Neurophysiology. 1994; 72:974-998. [PubMed: 7983549]

31. Blazquez PM, Hirata Y, Highstein SM. Chronic changes in inputs to dorsal Y neurons accompany VOR motor learning. J Neurophysiol. 2006; 95:1812-25. [PubMed: 16319196]

32. Medina JF, Nores WL, Ohyama T, Mauk MD. Mechanisms of cerebellar learning suggested by eyelid conditioning. Curr.Opin.Neurobiol. 2000; 10:717-724. [PubMed: 11240280]

33. Christian KM, Thompson RF. Neural substrates of eyeblink conditioning: acquisition and retention. Learn Mem. 2003; 10:427-55. [PubMed: 14657256]

34. Krauzlis RJ. Population coding of movement dynamics by cerebellar Purkinje cells. Neuroreport. 2000; 11:1045-50. [PubMed: 10790880]

35. Krauzlis RJ, Lisberger SG. Simple spike responses of gaze velocity Purkinje cells in the floccular lobe of the monkey during the onset and offset of pursuit eye movements. J Neurophysiol. 1994; 72:2045-50. [PubMed: 7823119]

36. Shidara M, Kawano K, Gomi H, Kawato M. Inverse-dynamics model eye movement control by Purkinje cells in the cerebellum. Nature. 1993; 365:50-2. [PubMed: 8361536]

37. Kahlon M, Lisberger SG. Changes in the responses of Purkinje cells in the floccular complex of monkeys after motor learning in smooth pursuit eye movements. J Neurophysiol. 2000; 84:294560. [PubMed: 11110823]

38. Lisberger SG, Fuchs AF. Role of primate flocculus during rapid behavioral modification of vestibuloocular reflex. II. Mossy fiber firing patterns during horizontal head rotation and eye movement. J Neurophysiol. 1978; 41:764-77. [PubMed: 96226] 
39. Miles FA, Fuller JH, Braitman DJ, Dow BM. Long-term adaptive changes in primate vestibuloocular reflex. III. Electrophysiological observations in flocculus of normal monkeys. J Neurophysiol. 1980; 43:1437-76. [PubMed: 6768853]

40. Highstein SM. Synaptic linkage in the vestibulo-ocular and cerebello-vestibular pathways to the VIth nucleus in the rabbit. Exp Brain Res. 1973; 17:301-14. [PubMed: 4722119]

41. Ron S, Robinson DA. Eye movements evoked by cerebellar stimulation in the alert monkey. J Neurophysiol. 1973; 36:1004-22. [PubMed: 4202613]

42. Wolpert, D.; Flanagan, J. Handbook of Brain Theory and Neural Networks. MA, A., editor. MIT Press; Cambridge, MA: 2002. p. 1020-1023.

43. Kawato M, Furukawa K, Suzuki R. A hierarchical neural-network model for control and learning of voluntary movement. Biol Cybern. 1987; 57:169-85. [PubMed: 3676355]

44. Pouget A, Snyder LH. Computational approaches to sensorimotor transformations. Nat Neurosci. 2000; 3(Suppl):1192-8. [PubMed: 11127837]

45. Jordan M, Rumelhart D. Forward models: supervised learning with a distal teacher. Cognitive Science. 1992; 16:307-354.

46. Fine MS, Thoroughman KA. Trial-by-trial transformation of error into sensorimotor adaptation changes with environmental dynamics. J Neurophysiol. 2007; 98:1392-404. [PubMed: 17615136]

47. Cheng S, Sabes PN. Calibration of visually guided reaching is driven by error-corrective learning and internal dynamics. J Neurophysiol. 2007; 97:3057-69. [PubMed: 17202230]

48. Jirenhed DA, Bengtsson F, Hesslow G. Acquisition, extinction, and reacquisition of a cerebellar cortical memory trace. J Neurosci. 2007; 27:2493-502. [PubMed: 17344387]

49. Ramachandran R, Lisberger SG. Transformation of vestibular signals into motor commands in the vestibuloocular reflex pathways of monkeys. J Neurophysiol. 2006; 96:1061-74. [PubMed: 16760348]

50. Lisberger SG, Pavelko TA. Vestibular signals carried by pathways subserving plasticity of the vestibulo-ocular reflex in monkeys. J Neurosci. 1986; 6:346-54. [PubMed: 3485189] 

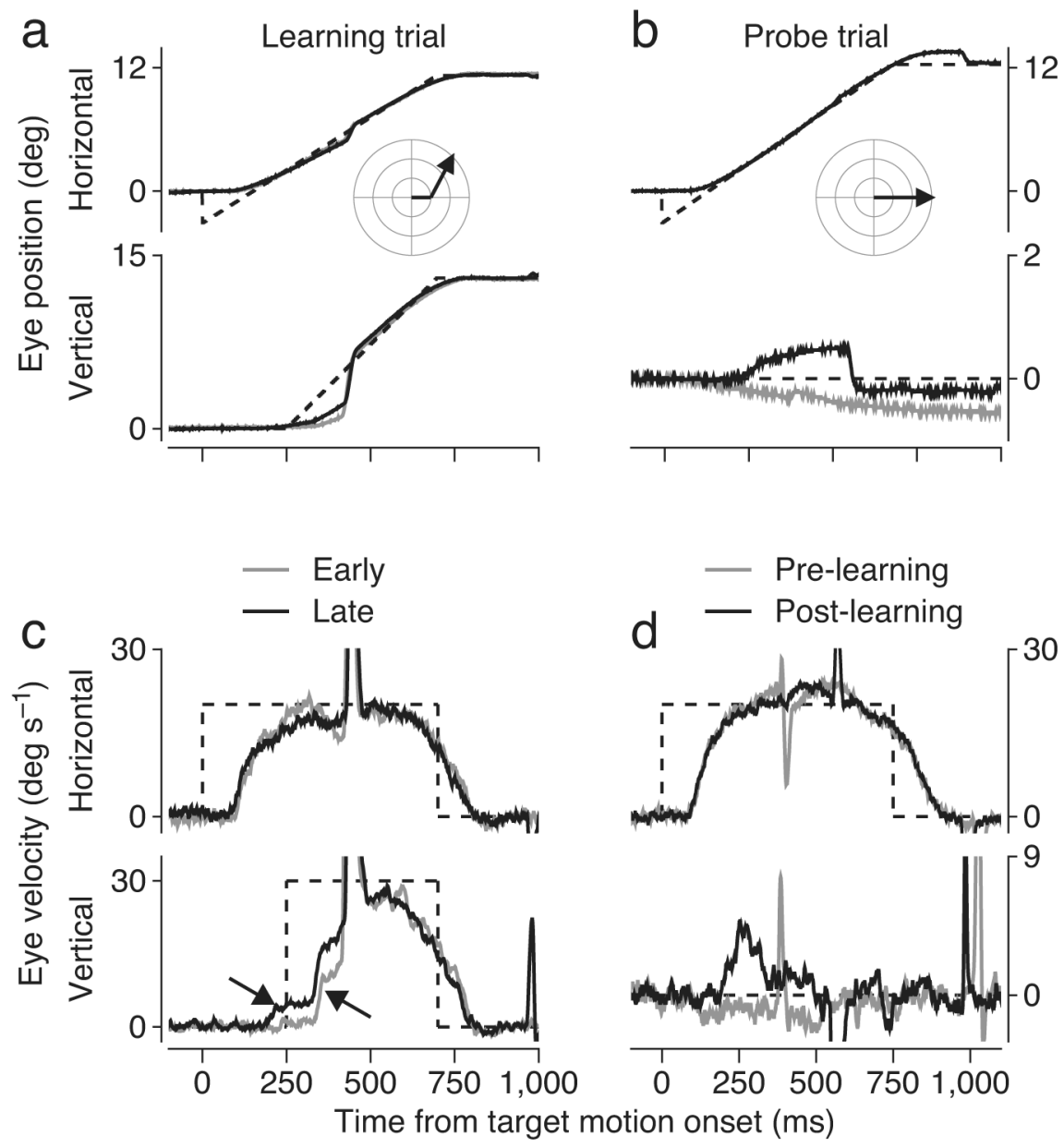

Figure 1.

Example trials showing the target motions used to instruct and probe directional learning in pursuit eye movements. Positive numbers represents motion to the right (horizontal plots) or up (vertical plots). Negative numbers represent motion to the left or down. a,c: Learning trials. Gray and black traces indicate data from representative trials early versus late in a learning block. b,d: Probe trials. Gray and black traces indicate data from representative trials before learning and after at least 100 learning trials. Dashed traces show target motion. Insets in $\mathbf{a}$ and $\mathbf{b}$ indicate the target motion in polar coordinates. The arrow pointing down and right in $\mathbf{c}$ indicates the learned response. The arrow pointing up and left indicates the hardwired visual response to the change in target direction. Note that the scales on vertical eye position and velocity are different in the probe versus the learning trials. 
a

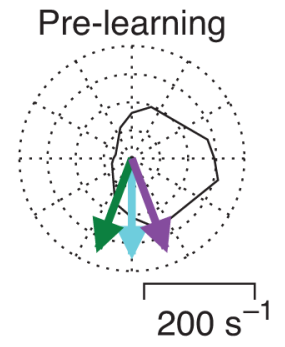

b Learning \#1

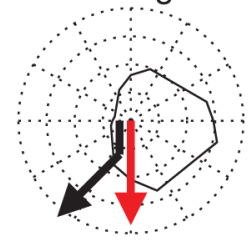

C Learning \#2

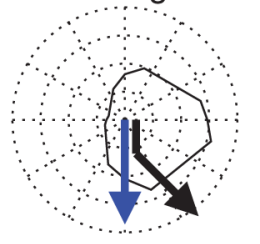

Eye velocity
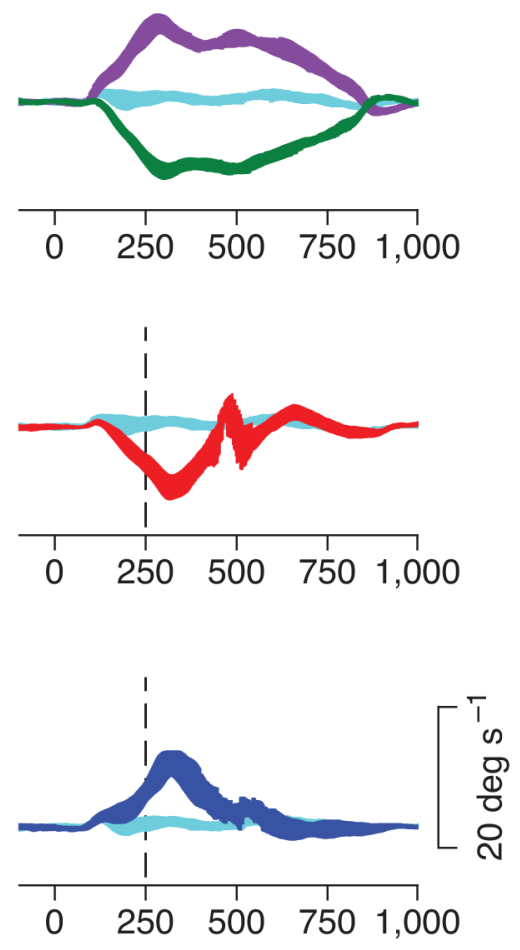

SS firing rate
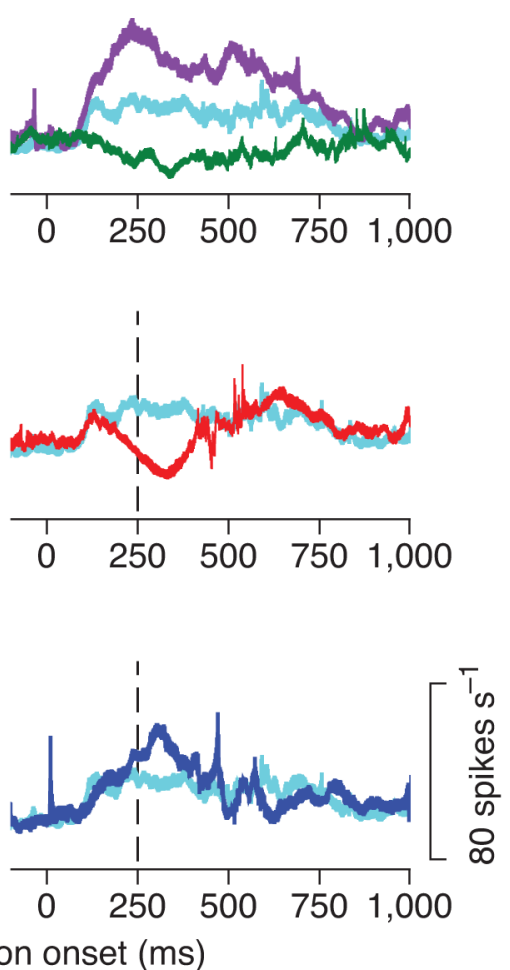

Figure 2.

Effects of learning on responses of one Purkinje cell. From top to bottom, the three rows show the control block, and the learning blocks in the off- and on-direction of the Purkinje cell. In the left column, the colored arrows show different target motions and the black continuous curve shows the direction tuning for the Purkinje cell. The middle and right columns show eye velocity and simple spike firing rate during probe trials. The colors of the traces refer to the different target trajectories indicated by the colored arrows in the polar plots. For ease of comparison, the light blue traces in $\mathbf{b}$ and $\mathbf{c}$ replot the pre-learning data for downward target motion from a. The ribbons show the mean \pm 1 SEM of eye velocity and firing rate. 
a

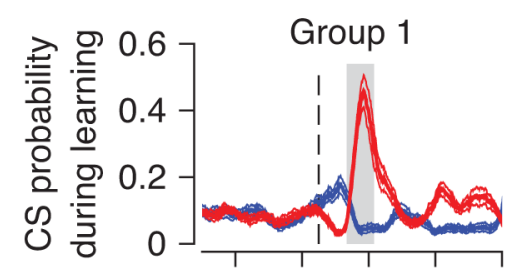

C

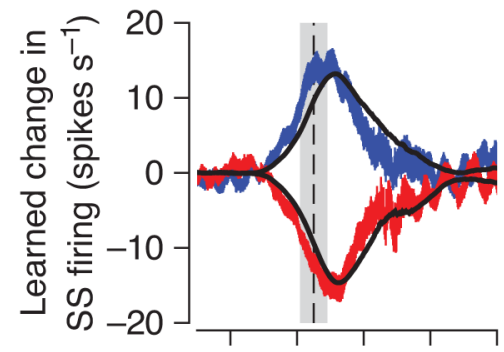

e

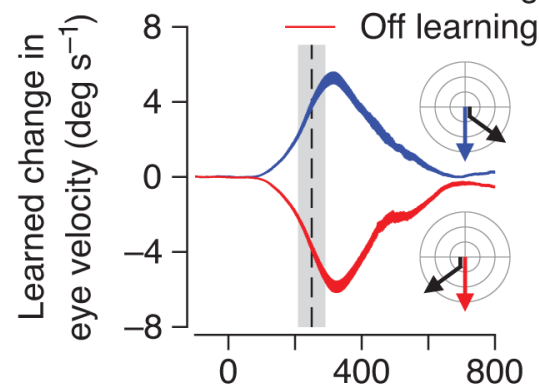

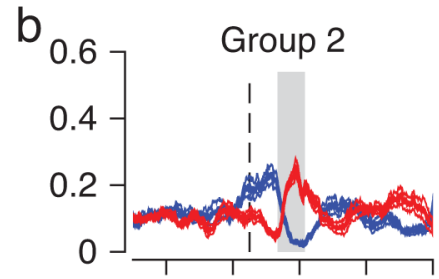

d

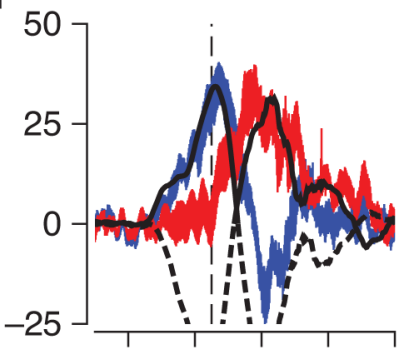

f

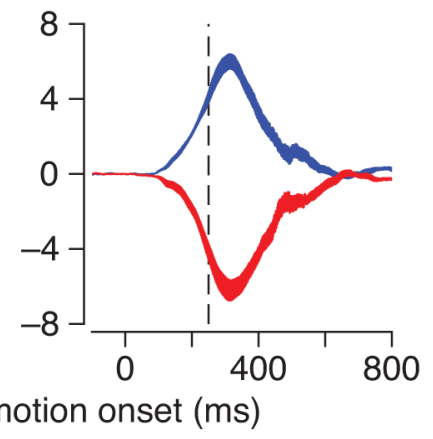

Figure 3.

Relationship between complex spike probability in learning trials and learned simple spike response in probe trials for group 1 and group 2 Purkinje cells. a, b: Probability of a complex spike during the first 50 learning trials in a learning block (measured in $100 \mathrm{~ms}$ bins). c, $\mathbf{d}$ : Learning-induced change in simple spike response, calculated as mean firing rate in probe trials after learning minus mean firing rate in pre-learning probe trials. In $\mathbf{c}$, the black traces repeat the eye velocity averages from $\mathbf{e}$. In $\mathbf{d}$, the black traces show eye acceleration for the eye velocity traces in $\mathbf{f}$, where the negative eye accelerations are shown as dashed traces. e, f: Learned eye movement, measured as the component of the eye velocity orthogonal to the direction of the target in probe trials. Blue and red traces indicate data for on-direction and off-direction learning, respectively. The ribbons show mean \pm 1 SEM. Vertical dashed lines show the time when the target changed direction in learning trials. Shaded areas show the analysis intervals for complex spike responses $(\mathbf{a}, \mathbf{b})$ and for simple spike responses and eye velocity $(\mathbf{c}, \mathbf{e})$. Data are averaged across 49 group 1 and 11 group 2 Purkinje cells. 


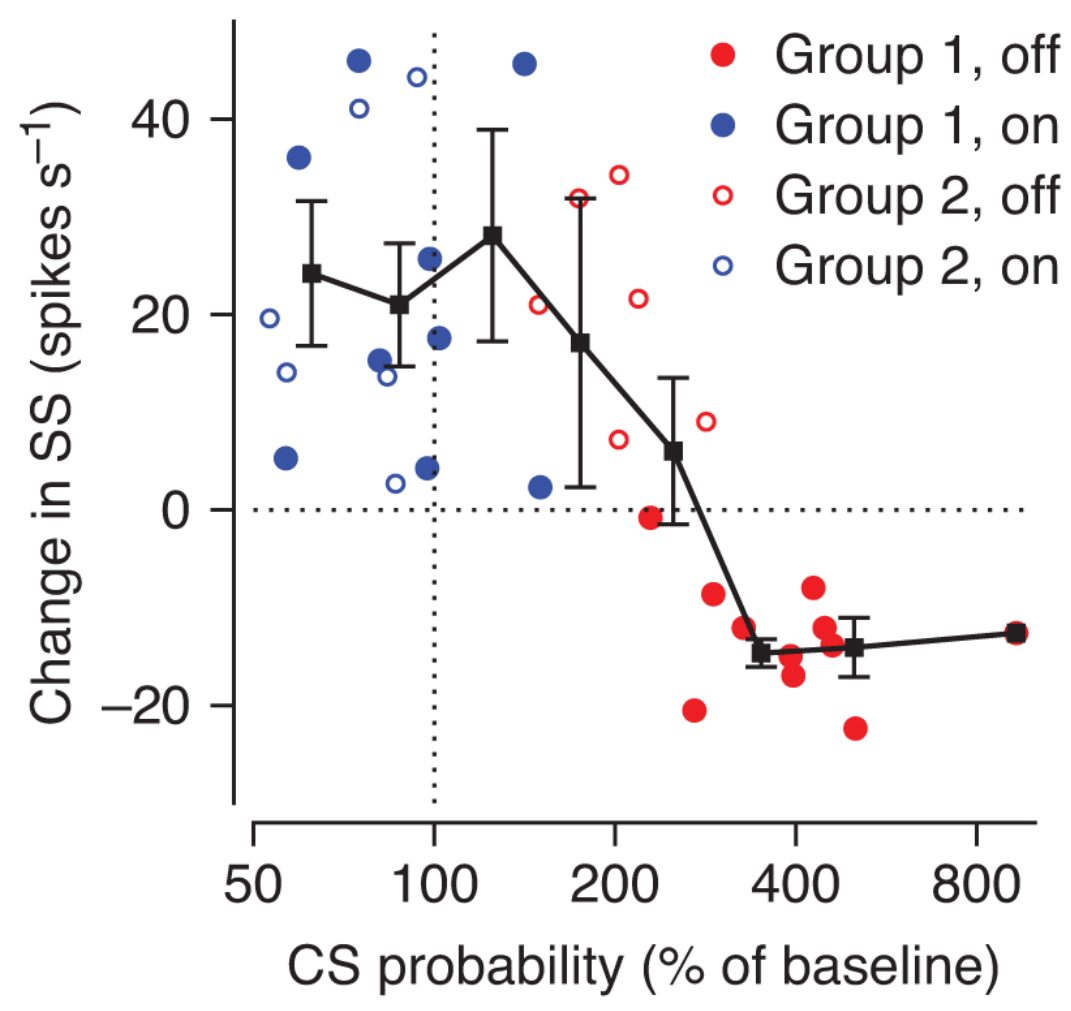

Figure 4.

Quantitative relationship between complex spike probability in learning trials and learned changes in simple spike activity in probe trials. Each point plots the results of one learning experiment on one Purkinje cell and shows averages across all the trials in the learning block. Analysis intervals for the complex spike probability and the learned change in simple spike firing are indicated in Figure 3. Filled and open symbols indicate data from group 1 $(\mathrm{n}=11)$ and group $2(\mathrm{n}=6)$ Purkinje cells; blue and red symbols indicate learning in the ondirection and off-direction of the Purkinje cell under study. 

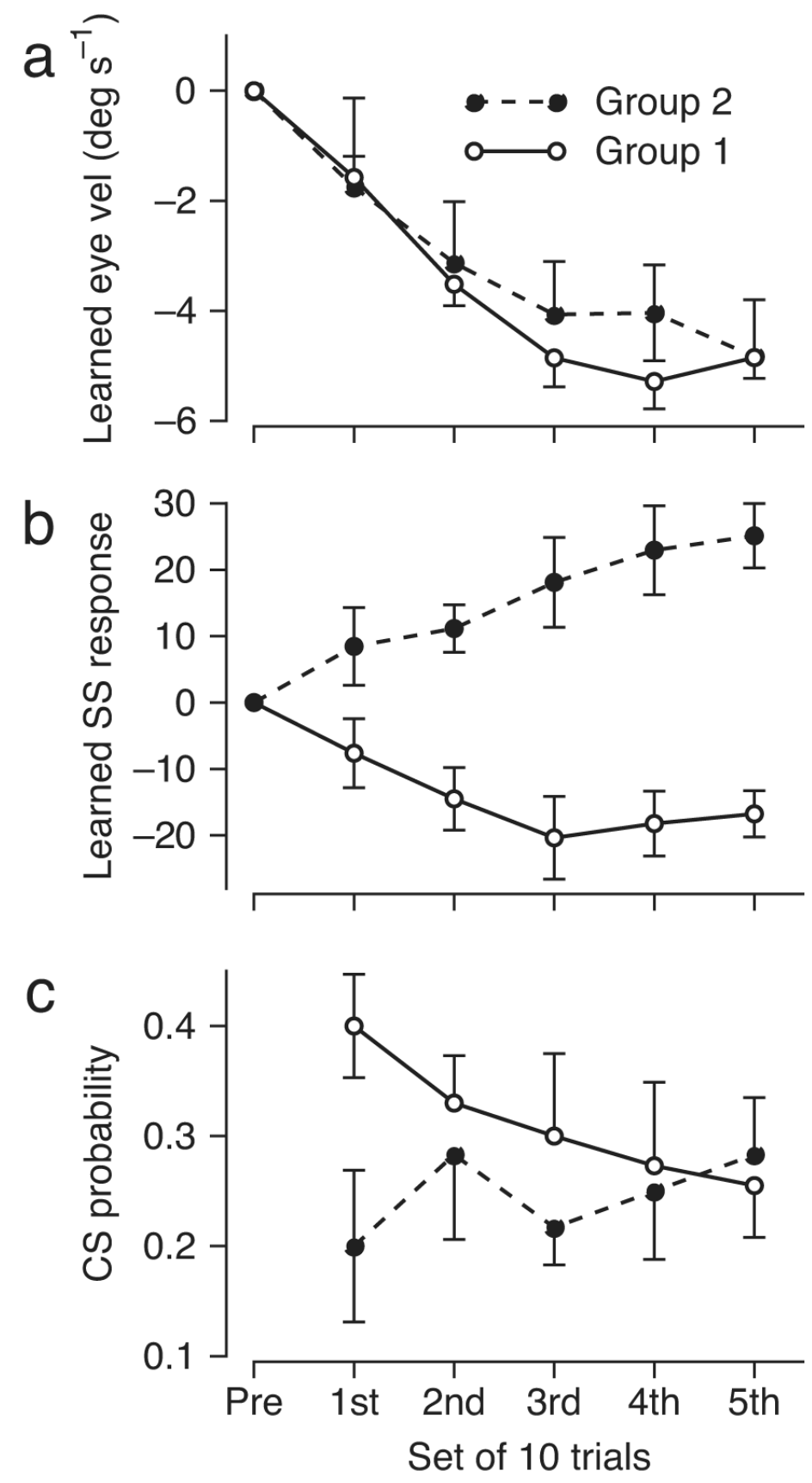

Figure 5.

Time courses of complex spike-related instructive signals and learned changes in eye velocity and simple spike responses. Each graph divides the first 50 learning trials for offdirection learning in sets of ten and plots the average response measure as a function of the set. a, b: Learned eye velocity and simple spike firing in probe trials. c: Probability of a complex spike in learning trials. Open and filled symbols show data from group $1(n=11)$ and group $2(n=6)$ Purkinje cells. 


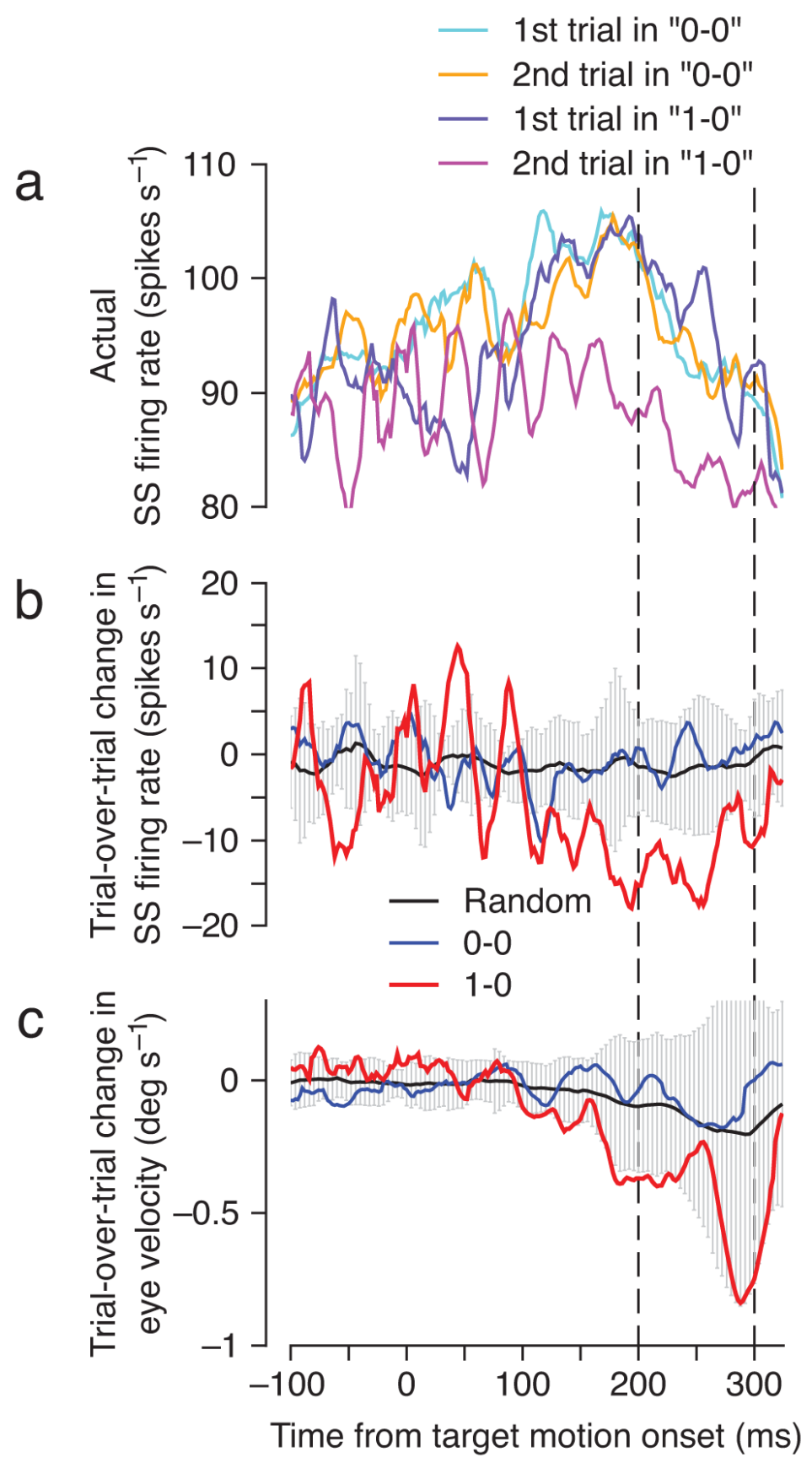

Figure 6.

Neural and behavioral learning after single complex spikes emitted during off-direction learning trials. All traces are plotted as a function of time, where the target started to move at $\mathrm{t}=0$, the target changed direction at $\mathrm{t}=250$, and the interval when learning was analyzed is bounded by the two vertical dashed lines. a: Actual simple spike firing rate on 1 st and 2 nd trials of each pair of successive learning trials computed separately for each one of 13 Purkinje cells in our sample and then averaged together. b, c: Average trial-over-trial change in simple spike firing (b) and eye velocity (c), for the " $0-0$ " sequences (blue) and the " $1-0$ " 
sequences (red). The black traces show the average change obtained by performing the trialover-trial subtraction on 200 randomly chosen sequences of 2 consecutive learning trials, and the gray envelope indicates $\pm 2 \mathrm{SD}$. 

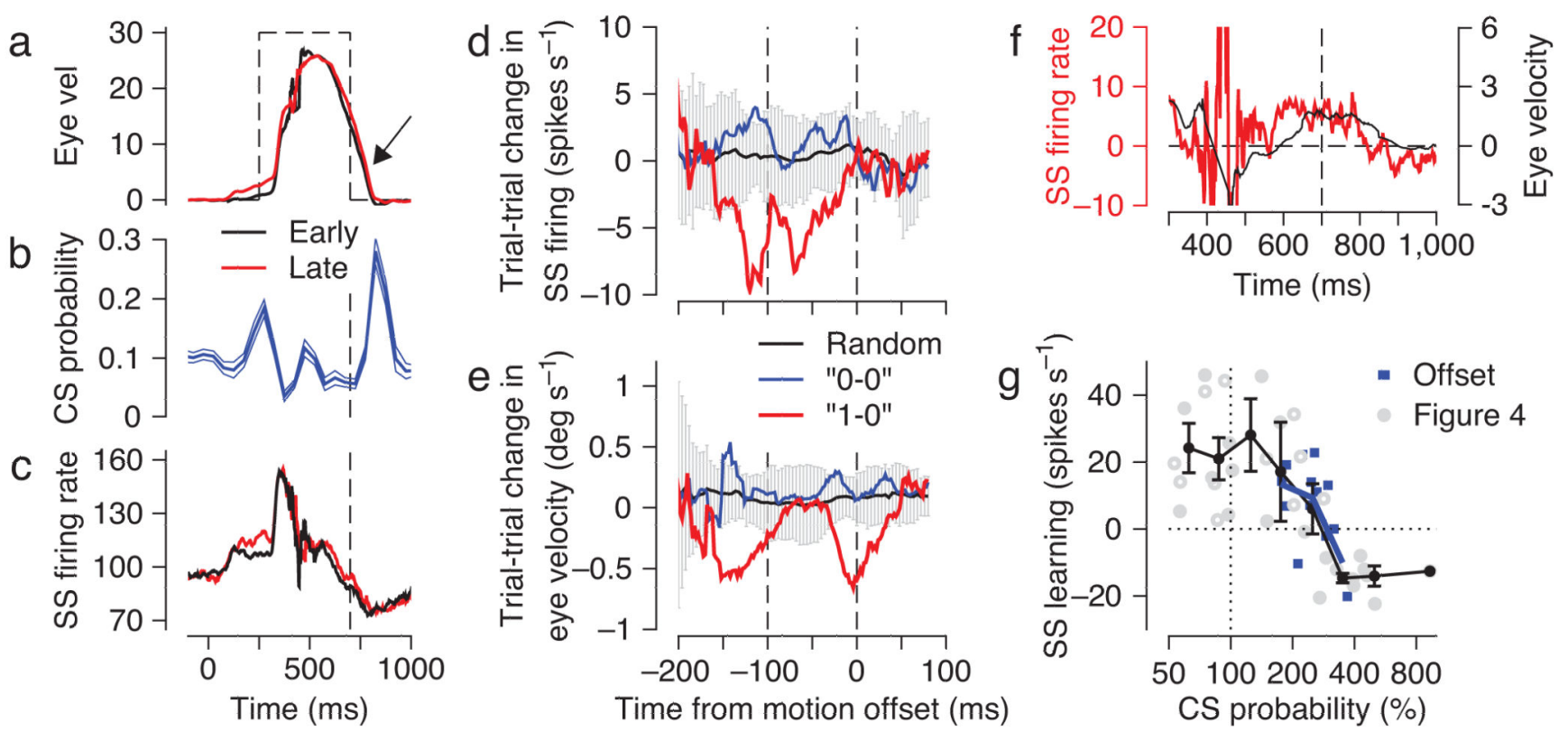

Figure 7.

Neural and behavioral learning after single complex spikes emitted at the offset of target motion in on-direction learning trials. a,c: Black and red traces show average eye velocity and simple spike firing in the first 10 learning trials and after 100 learning trials. In a, the black dashed trace shows target velocity. The arrow indicates the time when off-direction image motion was present because the eyes were moving in the on-direction and the target was stationary. b: Probability of a complex spike in the learning trials. Traces in a-c are averages across 13 Purkinje cells. Legend below a also applies to c. d,e; average trial-overtrial change in simple spike firing (d) and eye velocity (e), for the " $0-0$ " sequences (blue) and the " $1-0$ " sequences (red). The target ceased moving at $\mathrm{t}=0$, and the two vertical dashed lines bound the interval when learning was analyzed. The black traces show the average change obtained by performing the trial-over-trial subtraction on 200 randomly chosen sequences of 2 consecutive learning trials, and the gray envelope indicates \pm 2 SD. f: Red and black traces show learned changes in simple spike firing rate and eye velocity. Vertical dashed line indicates the time of the offset of target motion. Horizontal dashed line indicates zero. g: Quantitative relationship between complex spike probability and learned changes in simple spike activity. Blue symbols show data from the offset of target motion in ondirection learning experiments; gray symbols are replotted from Figure 4 showing data from the change in target direction in off-direction learning experiments. 